\title{
Symbolische Konstitutionalisierung und faktische Entkonstitutionalisierung: Wechsel von bzw. Änderungen in Verfassungstexten und Fortbestand der realen Machtverhältnisse
}

\author{
Von Marcelo Neves ${ }^{1}$
}

\section{Fortbestand und Transformation der Verfassungen in der herkömmlichen Verfassungslehre}

Die laufende Diskussion über den Fortbestand und die Transformation der Verfassungen geht von dem Modell der "normativen Verfassungen" aus. Diese werden als Verfassungen definiert, die in relevanter Weise die realen Machtverhältnisse regeln. In diesem Sinn verweist man auf einen ständigen Kern der Verfassungen, aus dem sie ihre eigene Identität beziehen. Hervorgehoben wird jedoch die Möglichkeit von Verfassungstransformationen, hinsichtlich welcher sich zwei grundlegende Arten erkennen lassen: Veränderungen der Verfassungsnormativität, die unmittelbar aus Änderungen in bzw. Wechsel von Verfassungstexten hervorgeht; und Wandlungen des normativen Sinns der Verfassung im Prozeß der Verfassungskonkretisierung.

Die unmittelbar aus Änderungen in bzw. Wechsel von Verfassungstexten hervorgehende Verfassungstransformation kann nach Maßgabe des in der Verfassung selbst festgesetzten Verfahrens erfolgen oder das Ergebnis eines Bruchs mit der bestehenden verfassungsrechtlichen Ordnung sein. Im ersten Fall wird die rechtliche Änderung der Verfassung in Brasilien gewöhnlich als "Verfassungsreform" bezeichnet. Einige Autoren versuchen die Reformen als Amendement und Revision zu klassifizieren, wobei erstere spezifischerer Natur wäre und die letztere den Anspruch hätte, den Verfassungstext in allgemeinerer Hinsicht zu tangieren. ${ }^{2}$ Diese Ausdrücke werden im positiven Verfassungsrecht allerdings nicht eindeutig verwendet. ${ }^{3}$ Strenggenommen wäre eine Unterscheidung zwischen (positivrecht-

Vgl. Jacques, Paulino, Curso de Direito Constitucional, 9. Aufl., Rio de Janeiro 1983 (1. Aufl.: 1956), S. 515; Menezes, Aderson de, Teoria Geral do Estado, Rio de Janeiro/São Paulo 1972, S. $226 \mathrm{f}$. 
licher) Verfassungsreform und faktischem oder rein politischem Verfassungswechsel angebracht. ${ }^{4}$ Es entsteht dann ein neuer Verfassungstext ohne eine konsistente Bindung an die aus dem vorgängigen Verfassungstext hervorgehende Normativität, vor allem ohne jegliche Begründung in vorgegebenen rechtlichen Verfahren. Normalerweise reduziert die Verfassungslehre diese Situation auf die revolutionäre verfassunggebende Gewalt - von der sich die staatsbegründende verfassunggebende Gewalt unterscheidet, ${ }^{5}$ die in Wahrheit revolutionäre Züge annimmt, wenn diese einen Bruch mit der vom Mutterland oktroyierten kolonialen rechtspolitischen Ordnung impliziert. Außer dem faktischen Verfassungswechsel, der aus einem radikalen Bruch mit dem Inhalt der vorhergehenden Rechtsordnung hervorgeht, gibt es jedoch auch die Möglichkeit, daß eine verfassunggebende Gewalt aus einem politischen Übergangsprozeß $\beta$ hervorgeht, ohne sich an das im vorhergehenden System vorgesehene Verfahren der Verfassungsänderung zu halten. Im Fall einer Revolution ereignet sich - über den Umstand der Durchführung gegen das vorgängig festgelegte Verfahren der Verfassungsänderung hinaus - ein politisch radikaler und heftiger Bruch mit dem Inhalt der vorhergehenden Rechtsordnung. Falls die verfassunggebende Gewalt ihren Ursprung in einem politischen Übergangsprozeß hat, geht man zwar auch nicht nach dem vorgängig festgelegten Verfahren vor, aber nach Maßgabe von politischen Vereinbarungen bzw. Kompromissen zwischen den Akteuren der alten und der neuen Ordnung im Hinblick auf den Inhalt der künftigen Verfassung; dann liegt politische Kontinuität und rechtliche Diskontinuität vor.

Hinsichtlich der Verfassungstransformationen, die aus dem Wandel des normativen Sinns des Verfassungstexts im Prozeß seiner Konkretisierung hervorgehen, sind zwei Möglichkeiten zu unterscheiden: erstens die Wandlungen, die sich aus der Verfassungs(text)auslegung durch das rechtsanwendende Organ ergeben; zweitens die Wandlungen, die sich aus der politischen Praxis ergeben, unabhängig von der hermeneutischen Tätigkeit angesichts des Verfassungstexts.

Zweifellos verändert sich der normative Sinn des Verfassungstexts zutiefst durch die Variierung der Interpretation. ${ }^{6}$ Die Hermeneutik hat unterstrichen, da $\beta$ die Interpretation bei der

21 ff.; Ferreira, Pinto, Princípios Gerais do Direito Constitucional Moderno, Bd. 1, 6. Aufl., São Paulo 1983, S. $116 \mathrm{f}$.

4

Vgl. z.B. Grimm, Dieter, Die Zukunft der Verfassung, Frankfurt am Main 1991, S. 117.

5

Vgl. Vanossi, Jorge Reinaldo A., Teoría Constitucional I - Poder Constituyente: fundacional, revolucionario, reformador, Buenos Aires 1975, S. $136 \mathrm{f}$.

6

Vgl. in unterschiedlichsten Perspektiven Kelsen, Hans, Reine Rechtslehre, 2. Aufl., Wien 1960; Smend, Rudolf, Verfassung und Verfassungsrecht (1928), in: ders., Staatsrechtliche Abhandlungen und andere Aufsätze, 2. Aufl., Berlin 1968, S. 119-275, S. 236; Ehrlich, Eugen, Grundlegung der Soziologie des Rechts, 3. Aufl., Berlin 1967 (unveränderter Nachdruck der 1. Aufl. 1913), S. 295; Ross, Alf, Lógica de las normas, spanische Übers. V. José Hierro, Madrid 1971, S. 111 f. u. S. 130; Miranda, Pontes de, Sistema de Ciência Positiva do Direito, Bd. I: Introdução à Ciência 
Konstruktion des normativen Sinns von Rechtstexten vom geschichtlich-sozialen Kontext bedingt ist, auf den jene appliziert werden. ${ }^{7}$ Solch eine semantische Variation des Sinns im Raum und in der Zeit kann nicht von den pragmatischen Bedingtheiten, d.h. von den Interessen, Erwartungen und Werten gelöst werden, die im Moment der Rechts(text)auslegung zur Sprache kommen. ${ }^{8}$ Dies gilt nicht nur in bezug auf die offiziellen Organe der konkretisierenden Rechts(text)auslegung ("Verfassungsinterpreten im engeren Sinn"), sondern auch in bezug auf die Öffentlichkeit, insofern sie ihre Erwartungen an den Verfassungstext heranträgt. ${ }^{9}$ Aus diesem Grund ist die radikale "originalistische Lehre" aus den USA, die eine Rückkehr zum Sinn vertritt, den die Mitglieder der Constitutional Convention von Philadelphia dem Verfassungstext von 1787 zuschrieben, unhaltbar. ${ }^{10}$ Man bemerkt vor allem am nordamerikanischen System, was für eine ausgezeichnete Rolle der aus der rechtsprechenden Tätigkeit hervorgehende Verfassungswandel spielt. Aber auch im kontinentaleuropäischen System wird die Rolle der Verfassungsgerichte als verfassungskonstruierende und rekonstruierende Instanzen immer deutlicher.

Der Wandel der Verfassung im Prozeß ihrer Verwirklichung ergibt sich auch aus der nicht an die Tätigkeit der Rechtsauslegung/-anwendung gebundenen Verfassungspraxis. Die Art und Weise, wie sich die Machtverhältnisse konkret entwickeln, wie die obersten Staatsorgane handeln, wie die Bürger sich zum Staat und zueinander verhalten, können relevante Verfassungswandlungen implizieren. Es ist sowohl möglich, daß dies Änderungen des normativen Sinns des Verfassungstexts oder die Ausfüllung von "Verfassungslücken" zum Ergebnis hat, als auch, daß dies das Aufkommen einer Verfassungsnormativität, die sich marginal zum Verfassungstext verhält, oder einfach den Nichtgebrauch von gewissen Verfassungsbestimmungen (desuetudo) befördert. Außerdem ist diese Form der Verfassungswandlung typisch für gewohnheitsrechtliche Verfassungen, die durch keinen Verfassungstext vermittelt sind.

do Direito, 2. Aufl., Rio de Janeiro 1972, S. 99; Carbonnier, Jean, Sociologie juridique, Paris 1972, S. $150-152$.

Vgl. Gadamer, Hans-Georg, Wahrheit und Methode: Grundzüge einer philosophischen Hermeneutik, 6. Aufl., Tübingen 1990, S. $330 \mathrm{ff}$.

8

Neves, Marcelo, Teoria da inconstitucionalidade das leis, São Paulo 1988, S. 127 ff.; Edelman, Murray, The Symbolic Uses of Politics, Urbana / Chicago / Londres 1967, S. 141.

9

Häberle, Peter, Die offene Gesellschaft der Verfassungsinterpreten: Ein Beitrag zur pluralistischen und 'prozessualen' Verfassungsinterpretation, in: ders., Die Verfassung des Pluralismus: Studien zur Verfassungstheorie der offenen Gesellschaft, Königstein/Ts. 1980, S. 79-105 (zuerst in: JZ 1975, S. 297-305).

10

Vgl. hierzu Rakove, Jack N. (Ed.), Interpreting the Constitution: The Debate over Original Intent, Boston 1990. 


\section{Entkonstitutionalisierende Konkretisierung des Verfassungstexts als Erhaltung der realen Machtverhältnisse}

Wenn wir von Arten der Verfassungstransformation in Sinn der gängigen Verfassungslehre sprechen, gehen wir von den "normativen Verfassungen" aus, setzen wir "die normative Kraft der Verfassung"11 voraus, verstanden als Erwartungsorientierung und Verhaltenssteuerung in der Öffentlichkeit nach Maßgabe des normativen Verfassungsmodells. Im Falle der "nominalistischen Verfassungen", in denen ein radikaler Hiatus zwischen Verfassungstext und Verfassungswirklichkeit klafft, ${ }^{12}$ wird eine sorgfältigere Bewertung des Problems ihrer Transformationen durch Verfassungswandel, Verfassungsreform resp. Verfassunggebung verlangt. Einerseits führt die Entstellung des Verfassungstexts im Konkretisierungsproze $\beta$, ohne das Fundament verallgemeinerungsfähiger normativer Kriterien, dazu, daß die Anwendung der Semantik der "konstitutionellen Mutation" fragwürdig wird. In diesen Situationen ist die Rede von faktischer Entkonstitutionalisierung oder entkonstitutionalisierender Konkretisierung angebracht. Andererseits wird das Problem komplexer, wenn wir die hypertroph symbolische Funktion der Änderungen in bzw. Wechsel von Verfassungstexten betrachten. Die "konstitutionelle Mutation" wird dann zum Topos der symbolischen Konstitutionalisierung.

Die Entkonstitutionalisierung, auf die wir uns hier beziehen, ist nicht diejenige, die im Zusammenhang der weitergesteckten Diskussion über Entrechtlichung diskutiert wird. ${ }^{13}$ Es handelt sich nicht um die Reduktion der konstitutionalisierbaren Materien und Beziehungen, die also durch Verfassungsnormen geregelt werden könnten. Dies ist ein typisches Problem der zentralen Länder, in denen die "normativen Verfassungen" zu einer radikalen Verrechtlichung der Öffentlichkeit geführt haben ${ }^{14}$ und so die Entstehung von entrechtlichenden und entkonstitutionalisierenden Gegentendenzen ermöglichten. Das Problem der

Hesse, Konrad, Die normative Kraft der Verfassung, in: ders., Ausgewählte Schriften, hrsg. v. P. Häberle u. A. Hollerbach, Heidelberg 1984, S. 3-18.

Vgl. Loewenstein, Karl, Verfassungslehre, dt. Übers. v. Rüdiger Boerner, 3. Aufl., Tübingen 1975, S. 151-57; ders., Gedanken über den Wert von Verfassungen in unserem revolutionären Zeitalter, in: Arnold J. Zurcher (Hrsg.), Verfassungen nach dem Zweiten Weltkrieg, dt. Übers. v. Ebba Vockrodt, Meisenheim am Glan 1956, S. 210-246, S. 222-225; Neves, Marcelo, Verfassung und Positivität des Rechts in der peripheren Moderne: Eine theoretische Betrachtung und eine Interpretation des Falls Brasilien, Berlin 1992, S. 91 ff.; ders., A Constitucionalização Simbólica, São Paulo 1994, S. 95-99.

Vgl. Voigt, Rüdiger (Hrsg.), Gegentendenzen zur Verrechtlichung (Jahrbuch für Rechtssoziologie und Rechtstheorie 9), Opladen 1983.

14

Vgl. Voigt, Rüdiger, Verrechtlichung: Analysen zu Funktion und Wirkung von Parlamentalisierung, Bürokratisierung und Justizialisierung sozialer, politischer und ökonomischer Prozesse, Königstein 1980; Habermas, Jürgen, Theorie des Kommunikativen Handelns, 2. Aufl., Frankfurt am Main 1982, S. 522 ff.; Werle, Raymund, Aspekte der Verrechtlichung, in: Zeitschrift für Rechtssoziologie 3, 1982, S. 2-13. 
faktischen Entkonstitutionalisierung in den peripheren Ländern mit "nominalistischen Verfassungen" bezieht sich auf die semantische Entwertung des Verfassungstexts im Prozeß seiner Konkretisierung. In diesem Zusammenhang entsteht nicht ein verallgemeinertes konsistentes Verhältnis von den verfassungsauslegenden/-anwendenden Tätigkeiten und den politischen Praxen der staatlichen Organe und der Bürger mit dem normativen Muster des Verfassungstexts. Man kann dann nicht von einer "kongruenten Generalisierung von normativen Erwartungen" ${ }^{15}$ reden, die auf der Grundlage des Verfassungstexts strukturiert und operationalisiert ist. Es ist also keine pluralistische Öffentlichkeit vorhanden, die verfassungsmäßig integriert wäre. Die rechtsnormative Konkretisierung des Verfassungstexts wird auf permanente und verallgemeinerte Weise vom wirtschaftlichen, politischen, familiären Druck oder vom Druck der "guten Beziehungen" usf. blockiert (nicht einfach bedingt), was im Gegensatz zur binären Codierung im Rahmen der Auffassung der autopoietischen Systeme ${ }^{16}$ den Zusammenbruch der operationalen Autonomie des Rechtssystems und eine selbst- und fremddestruktive Vermischung des rechtlichen, wirtschaftlichen, politischen, familialen, "beziehungsmäßigen" und anderer Codes zur Folge hat. Entkonstitutionalisierung bedeutet also Entrechtlichung infolge der Zerbrechlichkeit des rechtlichen Codes in seiner Unfähigkeit zur kongruenten Generalisierung und Mangel an konsistenter Autonomie bzw. Identität einer Rechtssphäre. ${ }^{17}$

Hier wären Verweise auf die Strukturierende Rechtslehre von Müller ${ }^{18}$ angebracht. Im Rahmen dieses theoretischen Paradigmas kann man beobachten, daß nicht nur die "Entscheidungsnorm" (individuelle Norm), sondern auch die "Rechtsnorm" (allgemeine Norm) im Konkretisierungsproze $\beta$ erzeugt wird. Der Verfassungstext stellt sich einerseits als "das

Vgl. Luhmann, Niklas, Ökologische Kommunikation: Kann die moderne Gesellschaft sich auf ökologische Gefährdungen einstellen?, Opladen 1986, S. 75 ff.; ders., Die Codierung des Rechtssystems, in: Rechtstheorie 17, 1986; ders., Das Recht der Gesellschaft, Frankfurt am Main 1993, S. $165 \mathrm{ff}$.

17 Neves, Marcelo, Do Pluralismo Jurídico à Miscelânea Social: O Problema da Falta de Identidade da(s) Esfera(s) de Juridicidade na Modernidade Periférica e suas Implicações na América Latina, in: Anuário do Mestrado em Direito, Recife: Universidade Federal de Pernambuco 1993, S. 313 357.

Müller, Friedrich (Hrsg.), Untersuchungen zur Rechtslinguistik: Interdisziplinäre Studien zu praktischer Semantik und strukturierender Rechtslehre in Grundfragen der juristischen Methodik, Berlin 1989; ders., Die Positivität der Grundrechte: Fragen einer praktischen Grundrechtsdogmatik, 2. Aufl., Berlin 1990; ders., Essays zur Theorie von Recht und Verfassung, hrsg. v. Ralph Christensen, Berlin 1990; ders., Strukturierende Rechtslehre, 2. Aufl., Berlin 1994; ders., "Juristische Methodik - ein Gespräch im Umkreis der Rechtstheorie", ein Interview mit Jan Möller aus Anlaß des Erscheinens der 5. Auflage des gleichnamigen Werkes von F. Müller, in: Verwaltungsrundschau 4, 1994, S. 133-136; vgl. Canotilho, J.J. Gomes, Direito Constitucional, 5. Aufl., Coimbra 1991, S. 208 ff. u. S. 221 ff. 
wichtigste Eingangsdatum" dieses Prozesses dar. ${ }^{19}$ Andererseits muß die konkretisierende Entscheidung konsistent dem Text "zurechenbar" sein, obgleich sie die unterschiedlichsten Inhalte auf weisen kann. ${ }^{20}$ Wenn aber eine entkonstitutionalisierende Konkretisierung vorkommt, gibt es kein konsistentes Verhältnis zwischen dem Text und den konkretisierenden Tätigkeiten. Der Verfassungstext ist eine entfernte Bezugsgröße für die staatlichen Akteure und für die Bürger, deren Praxis sich häufig am Rande des vertexteten Verfassungsmodells abspielt.

Der vorwiegend semantischen Blickrichtung von Müller ist hier die vornehmlich pragmati-

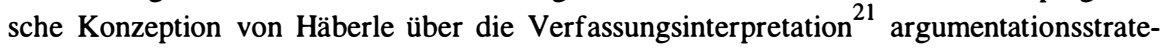
gisch hinzuzufügen. Neben den Interpreten im engeren Sinn (den für die Anwendung der Verfassung zuständigen Organen) bezieht Häberle die ganze Öffentlichkeit in den Prozeß der Verfassungsinterpretation ein: "In die Prozesse der Verfassungsinterpretation sind potentiell alle Staatsorgane, alle öffentlichen Potenzen, alle Bürger und Gruppen eingeschaltet" $^{22}$. Die normative Kraft der Verfassung wäre dann gewährleistet, wenn die pluralistische Öffentlichkeit in den Prozeß der Verfassungskonkretisierung eingebunden wäre. In dieser Perspektive müssen die unterschiedlichsten Interessen und Werte des Publikums vom Interpreten im engeren Sinn berücksichtigt werden. In systemtheoretischer Sprache könnte man hier sagen, daß die offiziellen Verfahren der Rechtsauslegung/-anwendung sich dann als Selektionsmechanismen der verschiedenen Erwartungen des Publikums in bezug auf den Verfassungstext darstellen. Diese Selektivität hätte eine die verfassungsnormativen Erwartungen stabilisierende und kongruent generalisierende Funktion. Im Fall der entkonstitutionalisierenden Konkretisierung ist jedoch erstens eine pluralistische Öffentlichkeit nicht vorhanden. Die exkludierenden Verhältnisse der Sub- und Überintegration in die Gesellschaft, besonders in das Rechtssystem, verhindern dessen Ausdifferenzierung ${ }^{23}$ und den Aufbau einer pluralistischen Öffentlichkeit, die aus Bürgern bestehen würde, die vom rechtlichen Standpunkt aus egalitär in die Gesellschaft integrierte Individuen wären. ${ }^{24}$ Das normative Handeln und Erleben des Unter- und Überbürgers führen zur Implosion der Verfassung selbst als rechtspolitisches Modell der Öffentlichkeit. In diesem Kontext muß

Müller, Friedrich, Essays zur Theorie von Recht und Verfassung (Fn. 18), S. 20; vgl. ibid., S. 127 u. S. 129; Jeand'Heur, Bernd, Gemeinsame Probleme der Sprach- und Rechtswissenschaft aus der Sicht der Strukturierenden Rechtslehre, in: Friedrich Müller, Untersuchungen zur Rechtslinguistik (Fn. 18), S. 17-26, S. 22.

Müller, Friedrich, Juristische Methodik (Fn. 18), S. 134.

Häberle, Peter, Die offene Gesellschaft der Verfassungsinterpreten (Fn. 9).

Ibid., S. 80.

Anders Luhmann, Niklas, Das Recht der Gesellschaft (Fn. 16), S. 582 ff.

Vgl. Neves, Marcelo, Verfassung und Positivität des Rechts (Fn. 12), S. 94 ff. und 155 ff.; ders., Entre Subintegração e Sobreintegração: A Cidadania Inexistente, in: Revista Acadêmia, Bd. LXXV (Recife: Universidade Federal de Pernambuco) 1992, S. 77-103. 
der Verweis auf die Öffentlichkeit durch die Beobachtung spezifiziert werden, daß es sich um eine auf bestimmte Gruppen und Organisationen "eingeschränkte Öffentlichkeit" handelt. Dabei fehlt der diskursethischen Konzeption der "privaten und öffentlichen Autonomie" (Menschenrechte und Volkssouveränität) ${ }^{25}$ also empirische Relevanz. Und mit dem Problem der unzureichenden Konstruktion der Öffentlichkeit in den Fällen der entkonstitutionalisierenden Konkretisierung hängt der Mangel an angemessener Selektivität seitens der offiziellen Verfahren der Verfassungsauslegung/-anwendung angesichts der politischen Erwartungen von Individuen und Gruppen zusammen. Diese Erwartungen sind nicht nur weitgehend verfassungsmarginal oder verfassungsdestruktiv; auch die Verfahren führen oft zur "kasuistischen" Verzerrung des normativen Sinnes des Verfassungstexts. Eine solche Situation bedeutet keine Dekonstruktion des Verfassungstexts in postmoderner Perspektive, sondern eher eine destruktive Praxis gegenüber seinen möglichen normativen Sinngehalten.

Die entkonstitutionalisierende Konkretisierung muß nicht auf der Grundlage der klassischen Dichotomie "Formale Verfassung versus materiale Verfassung" verstanden werden. Die politischen, wirtschaftlichen und "beziehungsmäßigen" Blockierungen des Prozesses der rechtsnormativen Konkretisierung des Verfassungstexts implizieren dann keine kongruente Generalisierung von normativen Erwartungen in bezug auf die grundlegenden politischen Verhältnisse. Es gibt eine partikularistische und diffuse Tendenz, welche sogar das Zustandekommen materialer Verfassungsrechtlichkeit, die verallgemeinerungsfähige normative Kriterien erfordern würde, verhindert. Und das bedeutet, wie ich schon vorher bemerkt habe, eine selbst- und fremddestruktive Vermischung von Verhaltenscodices, welche sogar die Autonomie/Identität der Rechtssphäre(n) trifft. Faktische Entkonstitutionalisierung erscheint also als Hauptform der Entrechtlichung des Konkretisierungsprozesses.

Die entkonstitutionalisierende Konkretisation im Rahmen der "nominalistischen Verfassungen" der peripheren Länder, mit besonderer Hervorhebung Brasiliens, wirkt im Sinne einer Erhaltung des sozialen status quo. Sie dient der Beibehaltung der realen Machtstrukturen, entgegen dem Textmodell der Verfassung, dessen relevante Verwirklichung tiefgreifende soziale Veränderungen nach sich ziehen würde. Gegen die im Verfassungstext ausgedrückten Veränderungsindizien setzen sich reale Machtverhältnisse mit dem Anspruch auf Verewigung durch, obgleich sie unstrukturiert sind und den Staat als Institution entstrukturieren. Es fragt sich somit, welches der Sinn der Ausarbeitung von Verfassungstexten unter derartigen Umständen ist. kratischen Rechtsstaats, Frankfurt am Main 1992, S. 112ff. 


\section{Wechsel von bzw. Änderungen in Verfassungstexten als symbolische Konstitutionalisierung}

Das Problem der häufigen Änderungen in bzw. Wechsel von Verfassungstexten ohne relevante rechtsnormative Resonanz in den Machtverhältnissen wird komplizierter, wenn wir die Diskussion über symbolische Konstitutionalisierung mit einbeziehen. ${ }^{26}$ Die entkonstitutionalisierende Konkretisierung ist der symbolischen Konstitutionalisierung innewohnend. Aber diese unterscheidet sich besonders durch ihre positive Bedeutung: die hypertroph politisch-ideologische Funktion des Textmodells der Verfassung.

Die Diskussion um die symbolische Konstitutionalisierung entwickelt sich im Ausgang von der Debatte über symbolische Gesetzgebung. ${ }^{27}$ Die Zweideutigkeit selbst der Termini 'Symbol', 'symbolisch' und 'Symbolismus' erschwert schon eine semantische Abgrenzung dieses Ausdrucks ${ }^{28}$. Vielleicht kann man eine Analogie zum Symbolismuskonzept Freuds vermuten, sofern dort zwischen latenter und manifester Bedeutung unterschieden ${ }^{29}$ und im Hinblick auf die symbolische Gesetzgebung behauptet wird, daß die latente über die manifeste Funktion vorherrscht. ${ }^{30}$

Aber die Frage der symbolischen Gesetzgebung verbindet sich gewöhnlich mit der Unterscheidung zwischen instrumentellen, expressiven und symbolischen Variablen im Rahmen der Sozialwissenschaften. Die instrumentellen Variablen würden eine Zweck-Mittel-Relation beinhalten, den bewußten Versuch, objektive Ergebnisse durch Handeln zu erzielen. In der expressiven Haltung gibt es eine Vermengung von Handeln und Befriedigung des

Neves, Marcelo, A constitucionalização Simbólica (Fn. 12); ders., Verfassung und Positivität des Rechts (Fn. 12), S. 61-65 u. 104-106.

Kindermann, Harald, Symbolische Gesetzgebung, in: Dieter Grimm / Werner Maihofer (Hrsg.), Gesetzgebungstheorie und Rechtspolitik (Jahrbuch für Rechtssoziologie und Rechtstheorie 13), Opladen 1988, S. 222-245; ders., Alibigesetzgebung als symbolische Gesetzgebung, in: Rüdiger Voigt (Hrsg.), Symbole der Politik, Politik der Symbole, Opladen 1989, S. 257-273; Bryde, BrunOtto, Effektivität von Recht als Rechtsproblem, Vortrag gehalten vor der Juristischen Gesellschaft zu Berlin am 17. März 1993 (Schriftenreihe der Juristischen Gesellschaft zu Berlin, H. 135), Berlin / New York 1993, S. $12 \mathrm{ff}$; Noll, Peter, Symbolische Gesetzgebung, in: Zeitschrift für Schweizerisches Recht (N.F.) 100, 1981, S. 347-364; Hegenbarth, Rainer, Symbolische und instrumentelle Funktionen moderner Gesetze, in: Zeitschrift für Rechtspolitik 14, 1981, S. 202204.

Neves, Marcelo, A Constitucionalização Simbólica (Fn. 12), S. 11 ff.

Vgl. Freud, Vorlesungen zur Einführung in die Psychoanalyse (1916-17 [1915-16]), in: FreudStudienausgabe, Bd. I, Frankfurt am Main 1969, S. 33-445, S. 159-177; ders., Die Traumdeutung (1900), in: Freud-Studienausgabe, Bd. II, Frankfurt am Main 1972, S. 435-494.

30

Vgl. Aubert, Vilhelm, Einige soziale Funktionen der Gesetzgebung, in: Ernst E. Hirsch / Manfred Rehbinder (Hrsg.), Studien und Materialien zur Rechtssoziologie (Sonderheft 11 der Kölner Zeitschrift für Soziologie und Sozialpsychologie), Köln / Opladen 1967, S. 284-309. 
jeweiligen Bedürfnisses. Während die instrumentelle Handlung sich als "Konfliktvehikel" konstituiert, ist das expressive Handeln ein "Vehikel der Katharsis" ${ }^{31}$. Indem er sich von anderen Autoren distanzierte, die das Problem der symbolischen Politik aufgriffen, unterschied Gusfield das Symbolische nicht nur vom Instrumentellen, sondern auch vom Expressiven. ${ }^{32}$ Entgegen der expressiven Haltung und ähnlich der instrumentellen Handlung zeichnet die symbolische Haltung sich nicht durch die Unmittelbarkeit der Befriedigung der jeweiligen Bedürfnisse aus und hängt mit dem Problem der Lösung von Interessenkonflikten zusammen. ${ }^{33}$ Anders als die instrumentellen Variablen, ist die symbolische Haltung nicht auf eine lineare Zweck-Mittel-Relation hin orientiert und ist andererseits nicht durch einen direkten und manifesten Zusammenhang zwischen Bezeichnendem und Bezeichnetem gekennzeichnet, sondern unterscheidet sich durch ihren mittelbaren und latenten Sinn. ${ }^{34}$ Wie Gusfield richtig bemerkt hat, "[ähnelt] die Unterscheidung zwischen instrumenteller und symbolischer Handlung in vielen Aspekten dem Unterschied zwischen denotativem und konnotativem Diskurs" ${ }^{35}$. In der Denotation gibt es einen relativ klaren Zusammenhang zwischen Ausdruck und Inhalt; im instrumentellen Handeln gibt es entsprechend eine Ausrichtung des Verhaltens auf feste Ziele. In der Konnotation ist die Sprache mehrdeutiger; das symbolische Handeln ist konnotativ, sofern es einen mittelbaren und undeutlichen Sinn erhält, der zu seinem unmittelbaren und manifesten Sinn hinzutritt ${ }^{36}$, und behält ihm gegenüber die Oberhand.

Die Unterscheidung zwischen instrumenteller, expressiver und symbolischer Funktion ist selbstverständlich nur in analytischer Hinsicht möglich; in der Praxis der sozialen Systeme sind diese drei Variablen immer gegenwärtig. Wenn man jedoch behauptet, daß ein Handlungszusammenhang eine symbolische, instrumentelle oder expressive Funktion hat, bezieht man sich auf die Dominanz einer dieser Variablen, niemals auf ihre Ausschließlichkeit. In diesem Sinn verweist "symbolische Gesetzgebung" auf die Vorherrschaft oder gar, in bezug auf das Rechtssystem, auf die Hypertrophie der symbolischen Funktion der gesetzgeberischen Tätigkeit und ihres Produkts, des Gesetzes, vor allem auf Kosten der rechtsinstrumentellen Funktion.

Gus field, Joseph R., Symbolic Crusade: Status Politics and the American Temperance Movement, 2. Aufl. (1. Aufl. 1963), Urbana / Chicago 1986, S. 179.

Ibid., S. 77 ff.; anders Luhmann, Niklas, Legitimation durch Verfahren, Frankfurt am Main 1983, S. $224 \mathrm{f}$.

Gus field, Joseph R., Symbolic Crusade (Fn. 31), S. 183.

Gusfield, Joseph R., Moral Passage: The Symbolic Process in Public Designations of Deviance, in: Social Problems, Bd. 15, No. 2 (Detroit, Michigan: Society for the Study of Social Problems), 1967, S. 175-188, S. $176 \mathrm{f}$. 
Es geht hier nicht um Konzeptionen der symbolischen Politik ${ }^{37}$ oder des Rechts als eines Symbolismus ${ }^{38}$, welche die Analyse eines jeden politischen bzw. rechtlichen Systems auf seine "symbolische Wirksamkeit" reduzieren. Obwohl Recht und Politik immer eine symbolische Dimension haben, gibt es in beiden Systemen relevante instrumentelle Variablen. Selbst die normative Kraft der Gesetzgebung hängt von einer Kombination von instrumentellen und symbolischen Variablen ab. Und das ganze Rechtssystem funktioniert auf der Grundlage beider Variablen. Das Problem entsteht dann, wenn in der Gesetzgebung hypertroph symbolische Wirkungen zu Lasten ihrer instrumentell-normativen Wirksamkeit auftreten.

Während die symbolische Gesetzgebung nur spezifische Sektoren des Rechtssystems berührt, trifft die symbolische Konstitutionalisierung infolge der größeren Weite des materialen und personalen Geltungsbereichs des Verfassungsrechts den Kern des Rechtssystems und belastet seine ganze operative Struktur und sogar seine Autonomie/Identität. Hier wird nicht verkannt, daß auch die "normativen Verfassungen" eine symbolische Funktion ausüben, wie Burdeau ${ }^{39}$ und Edelman ${ }^{40} \mathrm{zu}$ Recht hervorgehoben haben, unter Berufung auf die europäische bzw. nordamerikanische Verfassungserfahrung; ebensowenig wird übersehen, daß die Unterscheidung zwischen "normativer Verfassung" und "symbolischer Verfassung" relativ ist und es sich "eher um zwei Endpunkte auf einer Skala als um eine Dichotomie" handelt. ${ }^{41}$ Die symbolische Funktion der "normativen Verfassungen" ist aber an ihre rechtlich-instrumentelle Relevanz gekoppelt, d.h. an einen hohen Grad von verallgemeinerter normativer Konkretisierung der Verfassungsbestimmungen. "Normative Verfassungen" fungieren nicht nur als symbolischer Ausdruck der "Konsistenz", "Freiheit", "Gleichheit", "Partizipation" usf. als kennzeichnender Elemente der auf der Verfassung beruhenden politischen Ordnung; es läßt sich nicht bestreiten, daß sie rechtlich einen hohen Grad an Verhaltenssteuerung und an Erwartungssicherung ${ }^{42}$ implizieren. Den jeweiligen Verfassungsbestimmungen entsprechen in größerem oder geringerem Ausmaß, aber immer

Edelman, Murray, The Symbolic uses of Politics (Fn. 8); ders., Political Language: Words That Succeed and Policies That Fail, New York / San Francisco / London 1977.

Arnold, Thurman W., The symbols of Government, New Haven 1935 (5. Aufl. 1948), bes. S. $33 \mathrm{ff}$., oder ders., El derecho como simbolismo, in: Vilhelm Aubert (Hrsg.), Sociología del Derecho, spanische Übersetzung von J.V. Roberts, Caracas 1971.

Burdeau, Georges, Zur Auflösung des Verfassungsbegriffs, in: Der Staat 1, 1962, S. 389-404, S. 398; vgl. ferner Massing, Otwin, Identität als Mythopoem. Zur politischen Symbolisierungsfunktion verfassungsgerichtlicher Spruchweisheiten, in: Rüdiger Voigt (Hrsg.), Politik der Symbole, Symbole der Politik, Opladen 1989, S. 235-256.

41 Edelmann, Murray, The Symbolic Uses of Politics (Fn. 8), S. 18 f.

Bryde, Brun-Otto, Verfassungsentwicklung: Stabilität und Dynamik im Verfassungsrecht der Bundesrepublik Deutschland, Baden-Baden 1982, S. 27.

42 Vgl. Luhmann, Niklas, Ausdifferenzierung des Rechts: Beiträge zur Rechtssoziologie und Rechtstheorie, Frankfurt am Main 1981, S. 73-91. 
auf gesellschaftlich relevante Weise, "kongruent generalisierte normative Erwartungen". Das "Symbolische" und das "Instrumentelle" wirken wechselseitig aufeinander, um die Konkretisierung der Verfassungsnormen zu ermöglichen. Die Verfassung funktioniert wirklich als reflexive Instanz eines geltenden und wirksamen Rechtssystems.

Im Fall der symbolischen Konstitutionalisierung folgt jedoch auf die Verfassunggebung keine verallgemeinerte rechtliche Normativität, keine umfassende normative Konkretisierung des Verfassungstexts. So wie wir schon in bezug auf die symbolische Gesetzgebung behauptet haben, ist das Unterscheidungsmerkmal auch die Hypertrophie der symbolischen Dimension zu Lasten der rechtlich-instrumentellen Verwirklichung der Verfassungsbestimmungen. Der positive Sinn der symbolischen Konstitutionalisierung ist an seine negative Eigenschaft gebunden, die schon im vorhergehenden Abschnitt erörtert worden ist. ${ }^{43}$ Seine Definition beinhaltet diese zwei Momente: einerseits besteht seine Funktion nicht darin, nach Maßgabe der rechtlichen Bestimmungen der jeweiligen Verfassungstexte die Verhaltensweisen zu steuern und die Erwartungen zu orientieren; andererseits antwortet sie auf konkrete politische Forderungen und Ziele. "Dies kann rhetorische Verbeugung vor bestimmten Werten (Demokratie, Frieden) sein. Es kann aber auch um Propaganda gegenüber dem Ausland gehen" ${ }^{44}$.

Wir befinden uns hier in der Sphäre des Ideologischen im Sinne von Habermas ${ }^{45}$ : "Illusionen, die mit der Macht gemeinsamer Überzeugungen ausgestattet sind, nennen wir ja Ideologien." Es handelt sich aber nicht um Ideologie als Verzerrung einer wesentlichen Wahrheit. $^{46}$ Im Fall der symbolischen Konstitutionalisierung besteht das Problem darin, daß ein Modell übermittelt wird, dessen Verwirklichung nur unter völlig anderen sozialen Bedingungen möglich wäre. Auf diese Weise verliert man an Transparenz hinsichtlich der Tatsache, daß die dem symbolischen Verfassungsmodell entsprechende soziale Situation nur vermittels einer gesellschaftlichen Umwälzung zur Wirklichkeit werden könnte. Oder die Verfassungsfigurine tritt als Ideal auf, das über die "Machthaber" und ohne Schaden für die privilegierten Gruppen verwirklicht werden soll, wobei dann die rhetorische Formel der "guten Absicht" der Verfassungsväter und der Regierenden im allgemeinen herangezogen wird. 47

Vgl. Villegas, Maurício García, La Constitución e su Eficacia Simbólica, in: Revista Universidad de Antioquia, Bd. LX, N. 225 (Medellin), 1991, S. 4-21, S. 20, mit Bezug auf die kolumbianische Erfahrung.

44

Bryde, Brun-Otto, Verfassungsentwicklung (Fn. 41), S. 28.

Habermas, Jürgen, Hannah Arendts Begriff der Macht, in: Jürgen Habermas, Philosophischpolitische Profile, Frankfurt am Main 1987, S. 228-248, S. 246.

46

Hierzu kritisch Luhmann, Niklas, Wahrheit und Ideologie: Vorschläge zur Wiederaufnahme der Diskussion, in: Der Staat 1, 1962, S. 431-448.

47 Vgl. Schindler, Dietrich, Verfassungsrecht und soziale Struktur, 4. Aufl., Zürich 1967, S. 66 f. 
Der "Scheinkonstitutionalismus" 48 impliziert unter diesen Bedingungen eine illusorische Darstellung in bezug auf die Verfassungswirklichkeit und dient eher der Immunisierung des politischen Systems gegen andere Alternativen. Durch ihn können nicht nur die Probleme und Beziehungen, die auf der Grundlage der jeweiligen Verfassungsbestimmungen normiert würden, unverändert fortdauern ${ }^{49}$, sondern kann auch der Weg der sozialen Änderungen in Richtung des proklamierten Verfassungsstaats blockiert werden. (Es ist aber darauf hinzuweisen, daß selbst die normativen Verfassungen die sozialen Probleme nicht direkt lösen, sondern ihre Lösung nur mittelbar beeinflussen können. ${ }^{50}$ ). Zum Diskurs der Macht gehört also die ständige Berufung auf die Verfassungsurkunde als normative Struktur der Gewährleistung der Grundrechte, der "Gewaltenteilung" und der demokratischen Wahl, und der rhetorische Rekurs auf diese Institutionen als Errungenschaften der Regierung bzw. des Staats und Beweise des Vorkommens von Demokratie im Lande. Die ideologisch aufgeladene Formel "demokratische Gesellschaft" wird von den mit "symbolischen Verfassungen" Regierenden so regelmäßig benutzt wie von ihren Kollegen unter "normativen Verfassungen", wobei unterstellt wird, daß es sich um dieselbe Verfassungswirklichkeit handelt. Daraus entspringt eine pragmatische Verzerrung der Verfassungssprache, die zwar einerseits die soziale Spannung vermindert und die Wege zur Veränderung der Gesellschaft verstellt, indem sie das System gegen andere Alternativen immunisiert, andererseits aber in Extremfällen zum Mißtrauen des Publikums gegen das politische System und die staatlichen Akteure führen kann. In diesem Sinn hat selbst die ideologische Funktion der symbolischen Konstitutionalisierung ihre Grenzen und kann die Situation sich widersprüchlicherweise in ihr Gegenteil verkehren, im Sinn einer Bewußtmachung des Auseinanderklaffens von politischer Handlung und konstitutionalistischem Diskurs.

Das Problem der symbolischen Konstitutionalisierung darf nicht mit der mangelnden Wirksamkeit einiger spezifischer Bestimmungen des Verfassungstexts verwechselt werden, selbst wenn in diesem Fall das Ausbleiben der normativen Konkretisierung mit der symbolischen Funktion verbunden ist. Die Existenz von Verfassungsbestimmungen mit rein symbolischer Wirksamkeit ist immer möglich, ohne daß daraus das Verfassungssystem in seinen Grundzügen kompromittiert ist. Wir sprechen von symbolischer Konstitutionalisierung, wenn das Problem des hypertroph politisch-ideologischen Funktionierens der sich auf die (den) Verfassung(stext) beziehenden Tätigkeit die Hauptpfeiler des verfassungsrechtlichen Gefüges ins Wanken bringt. Dies geschieht dann, wenn die grundlegenden Verfassungsinstitutionen - die Grundrechte, die "Gewaltenteilung" und die demokratische

Grimm, Dieter, Verfassung, in: Staatslexikon: Recht - Wirtschaft - Gesellschaft, hrsg. v. d. Görres-Gesellschaft, 7. Aufl., 5. Bd., Freiburg / Basel / Wien 1989, Sp. 633-643, Sp 634, oder ders., Die Zukunft der Verfassung (Fn. 4), S. 13.

49

Bryde, Brun-Otto, Verfassungsentwicklung (Fn. 41), S. $28 \mathrm{f}$.

50

Grimm, Dieter, Verfassung (Fn. 48), Sp. 638, oder ders., Die Zukunft der Verfassung (Fn. 4), S. 19. 
Wahl - weder in der Praxis der Staatsorgane noch im Verhalten und in den Erwartungen der Bevölkerung auf allgemeine Resonanz stoßen. Die symbolische Konstitutionalisierung wird jedoch vor allem in bezug auf das Prinzip der Gleichheit vor dem Gesetz, das die Verallgemeinerung des Codes 'Recht/Unrecht ${ }^{51}$, d.h. den Zugang der gesamten Bevölkerung zum Rechtssystem impliziert, gekennzeichnet werden. Man kann behaupten, daß die "Verfassungswirklichkeit" dann entgegen der Verallgemeinerung des Rechts, die aus dem in der Verfassung symbolisch-ideologisch proklamierten Gleichheitsprinzip hervorgehen würde, partikularistisch ist, einschließlich im Hinblick auf die Praxis der Staatsorgane. Dem symbolisch inkludierenden Verfassungstext steht die exkludierende "Verfassungswirklichkeit" gegenüber. Die Grundrechte, die "Gewaltenteilung", die demokratische Wahl und die Gleichheit vor dem Gesetz, die als Rechtsinstitute umfassend in der Verfassungssprache vorgesehen sind, werden in der Praxis des Konkretisierungsprozesses verzerrt, besonders im Hinblick auf die Generalisierung, sofern sie sich einer Filterung nach Maßgabe partikularistischer Kriterien politischer, wirtschaftlicher usf. Natur unterwerfen. In diesem Zusammenhang wäre es nur angemessen, von eingeschränkter, exkludierender, partikularistischer Normativität zu sprechen, die sich also gegen die verallgemeinerte und inkludierende Normativität wenden würde, die im Verfassungstext proklamiert ist. Aber die im Verfassungstext verkündigten "Rechtsinstitutionen" sind weiterhin relevant als symbolische Bezugsgrößen des Machtdiskurses.

Zum Abschluß möchte ich darauf hinweisen, daß hier das Symbolische nicht mit dem Ideologischen verwechselt wird. ${ }^{52}$ Es ist unbestreitbar, daß das Symbolische eine relevante Rolle bei der Bewußtmachung spielen und damit "emanzipatorische" Wirkungen hervorbringen kann. Lefort verweist auf die Relevanz der "legalen" Erklärungen der "Menschenrechte" im demokratischen Rechtsstaat, deren symbolische Funktion zur Eroberung und Ausweitung dieser Rechte geführt habe. ${ }^{53}$ Aber im Fall der symbolischen Konstitutionalisierung, insbesondere als Alibi-Konstitutionalisierung ${ }^{54}$ ereignet sich eher eine Überschneidung zwischen dem Symbolischen und dem Ideologischen als ein kritischer Prozeß der Bewußtmachung von Rechten, eben in dem Maße, in welchem man das politische System gegen andere Möglichkeiten immunisiert und die Lösung der Probleme auf eine ferne Zukunft vertagt.

51

Luhmann, Niklas, Die Codierung des Rechtssystems (Fn. 16); ders., Das Recht der Gesellschaft (Fn. 16), S. 165 ff.

52 In anderem Zusammenhang vgl. Bryde, Brun-Otto, Effektivität von Recht als Rechtsproblem (Fn. 27), S. 16.

Lefort, Claude, Droits de l'homme et politique, in: ders., L'Invention Démocratique: Les limites de la domination totalitaire, Paris 1981, S. 45-83, S. 67 ff. u. S. 82.

54 Neves, Marcelo, A Constitucionalização Simbólica (Fn. 12), S. 92 ff.; vgl. Kindermann, Harald, Alibigesetzgebung (Fn. 27). 
Das Verständnis der symbolischen Konstitutionalisierung als Alibi zugunsten der herrschenden politischen Akteure und zu Lasten der Verfassungskonkretisierung findet Rückhalt in Brydes ${ }^{55}$ Beobachtungen über die afrikanische Erfahrung: Entgegen den "normativen Verfassungen" gründen sich die "symbolischen Verfassungen" hauptsächlich "auf die (internen oder externen Bedürfnissen entsprechenden) Ansprüche der Führungselite an die symbolische Darstellung ihrer Staatsordnung". Aus ihnen entspringt keine reale Veränderung des Machtprozesses. Es gibt zumindest eine rhetorische Vertagung der Verwirklichung des Verfassungsmodells auf eine ferne Zukunft, als ob dies ohne radikale Veränderungen der Machtverhältnisse und der sozialen Struktur möglich wäre.

\section{Abschließende Überlegungen}

Die Analyse, derzufolge die symbolische Konstitutionalisierung Änderungen in bzw. Wechsel von Verfassungsurkunden ohne den entsprechenden Wandel der realen zugrundeliegenden Gesellschaftsstrukturen impliziert und sogar als Illusionen aufbauender Mechanismus wirken kann, mag zu vereinfachenden Interpretationen führen, nach denen die Versuche zu durch Verfassungsreform bzw. Verfassunggebung vermittelten sozialen Wandlungen völlig nutzlos wären. Die hypertroph symbolische Funktion des Verfassungstexts bezieht sich jedoch nicht nur auf die "legitimierende" Rhetorik der Staatsregierenden. Auch im politischen Diskurs der Kritiker des Herrschaftssystems spielt die Berufung auf die im Verfassungstext proklamierten Werte eine relevante symbolische Rolle. So ist beispielsweise die politisch-soziale Rhetorik der "Menschenrechte" paradoxerweise umso intensiver, je geringer der Grad der normativen Konkretisierung des Verfassungstexts ist.

Obwohl die symbolische Konstitutionalisierung relevant im politischen Spiel ist, folgt auf sie nicht, besonders in der exkludierenden Struktur der brasilianischen Gesellschaft, eine "Massenloyalität", die einen effizienten Wohlfahrtsstaat voraussetzen würde. ${ }^{56}$ In dem $\mathrm{Ma} \beta$, in dem das Ausbleiben einer normativen Konkretisierung der Verfassungsurkunde und gleichzeitig der konstitutionalistische Machtdiskurs sich extrem ausweiten, steigt der Grad des Mißtrauens gegenüber dem Staat. Die Staatsgewalt wird diskreditiert. Die mangelnde Konsistenz der "Verfassungsordnung" führt zur Abnutzung des konstitutionalistischen Diskurses selbst der Kritiker des Herrschaftssystems. Auf die Demaskierung der konstitutionalistischen Farce folgt der Zynismus der Eliten und die Apathie des Publi kums. ${ }^{57}$ Eine solche Situation kann zur politischen Stagnierung führen. Es ist möglich, daß man im Zuge einer Reaktion auf den "Verfassungsrealismus" oder den "objektiven Idealis-

55

56

57

Bryde, Brun-Otto, Verfassungsentwicklung (Fn. 41), S. 29

Vgl. Neves, Marcelo, A Constitucionalização Simbólica (Fn. 12), S. 107 ff.

Vgl. Kindermann, Harald, Alibigesetzgebung (Fn. 27), S. 270, spezifisch in bezug auf die Alibigesetzgebung. 
mus" rekurriert, in Gegenposition zum existierenden "utopischen Idealismus". ${ }^{58}$ Wie jedoch die brasilianischen Erfahrungen des "instrumentellen Konstitutionalismus" von 1937 und 1964 gelehrt haben, wird der Rekurs auf diese autoritäre Semantik sicherlich nicht die "Versöhnung des Staats mit der nationalen Wirklichkeit" nach sich ziehen, sondern eher die exkludierende Identifizierung des staatlichen Rechtssystems mit den "Ideologien" und Interessen der eventuellen Machtinhaber. In diesem Fall werden diktatorische "gag rules" auferlegt werden, bei Vorenthaltung der für die symbolische Konstitutionalisierung typischen Möglichkeit der allgemeinen Kritik am Machtsystem.

Die symbolische Konstitutionalisierung darf deshalb nicht als ein Nullsummenspiel im politischen Kampf um die Ausweitung oder Einschränkung der citizenship interpretiert werden, als ob sie sich dem "konstitutionellen Instrumentalismus" der autokratischen Erfahrungen gleichstellen ließe. ${ }^{59}$ Solange weder demokratische noch diktatorische "gag rules" gelten ${ }^{60}$, ermöglicht der Kontext der symbolischen Konstitutionalisierung die Entstehung von sozialen Bewegungen und Organisationen, die sich kritisch für die Verwirklichung der im Verfassungstext feierlich proklamierten Werte engagieren und daher in den politischen Kampf um die Ausweitung der citizenship integriert sind. Wenn dem so ist, ist der Aufbau einer pluralistischen Öffentlichkeit möglich, die trotz ihrer Eingeschränktheit fähig ist, sich erfolgreich über die im Verfassungstext vorgesehenen demokratischen Verfahren zu artikulieren. Man kann allerdings nicht die Möglichkeit ausschließen, daß die Verwirklichung der in der Verfassungsurkunde formulierten demokratischen Werte einen Moment des Bruchs mit der etablierten Machtordnung voraussetzt. Und dies wird in dem $\mathrm{Ma} ß$ wahrscheinlicher, in dem die im Verfassungstext vorgesehenen demokratischen Verfahren im Laufe des Konkretisierungsprozesses verzerrt werden und sich nicht als staatliche Legitimationsmechanismen operationalisieren.

Vgl. Vianna, Oliveira, O Idealismo da Constituição, 2. Aufl., São Paulo / Rio de Janeiro / Recife / Porto Alegre 1939, bes. S. 7 ff. und S. $303 \mathrm{ff}$; Reale, Miguel, Momentos decisivos do constitucionalismo brasileiro, in: Revista de Informação Legislativa (Brasília), Jg. 20, N. 77, 1983, S. 57-68; Torres, Alberto, A organização nacional: Primeira parte, A Constituição, 3. Aufl., São Paulo 1978 (1. Aufl. 1914), S. $160 \mathrm{ff}$.

In anderem Sinn s. Loewenstein, Karl, Gedanken über den Wert von Verfassungen (Fn. 12), S. 224.

60 Neves, Marcelo, Verfassung und Positivität des Rechts (Fn. 12), S. 106 f.; ders., A Constitucionalização Simbólica (Fn. 12), S. 110 ff.; vgl. Holmes, Stephen, Gag Rules or the Politics of Omission, in: Jon Elster / $R$. Slagstad (Hrsg.), Constitutionalism and Democracy (Studies in Rationality and Social Change), Cambridge 1988, S. 19-58. 


\title{
Symbolical Constitutionalization and Effective De-Constitutionalization: Changes of and in Constitutional Documents and the Continuation of the Distribution of Power
}

\author{
By Marcelo Neves
}

The debate on constitutionalization and de-constitutionalization, even in the countries of the South, has concentrated on the situation formed by the normative constitutions of Northern America and of Western Europe, all of which are governed by the Rule of Law. The arguments of the debate reached Brazil and other peripheral (developing) countries almost totally unfiltered. Thus, there is a need to reconsider the questions with reference to the situation in modern-peripheral countries like Brazil.

The first part of the article focuses on traditional constitutional theory. The second part describes - on the basis of experiences in Brazil as a modern-peripheral country - the notion of effective de-constitutionalization, i.e. the practice of individuals, institutions and private organizations to distort and misuse the text of the constitution both in the political and legal sphere. In the light of this practice, many amendments to the Constitution of Brazil may be characterized as symbolical constitutionalization. In the final part the author describes the positive effects of symbolical constitutionalization on social change and indicates the limits to the practice of symbolical constitutionalization.

The article intends to initiate a new discussion about these constitutional developments in to-day Brazil.

\section{Gender Equality under the Japanese Constitution}

\section{By Annette Marfording}

The Japanese Constitution 1945 introduced the recognition and protection of gender equality. Equal rights for women were subsequently implemented in electoral, educational, employment and family laws. In 1986, the Equal Employment Opportunity Act came into force.

This article explores to what extent the Japanese government has fulfilled the constitutional mandate of gender equality, in particular, whether it has demonstrated a commitment to substantial rather than purely formal gender equality. The focus is on education, employment and family law, examined from a historical, legal, political, economic and cultural perspective. 